International Journal of Pure and Applied Mathematics

Volume 109 No. 2 2016, 451-457

ISSN: 1311-8080 (printed version); ISSN: 1314-3395 (on-line version)

url: http://www.ijpam.eu

doi: 10.12732/ijpam.v109i2.18

ijpam.eu

\title{
THE CONCEPT OF HIGHER ORDERS QUEUES IN THE QUEUING THEORY
}

\author{
Anton Titovtsev \\ Kazan National Research Technological University \\ 68, K. Marksa, Kazan, 420015 RUSSIA
}

\begin{abstract}
The paper considers various types of queues arising in queuing systems. The concept of an N-th order queue is introduced; the calculation algorithm of the first and second moments of main discrete and continuous random variables characterizing behavior of higher orders queues in queuing systems of the mixed type is presented.
\end{abstract}

AMS Subject Classification: 60K25

Key Words: physical queue, real queue, quality of service (QoS), queuing system (QS)

\section{Main Definitions}

An N-th order queue will be called the queue calculated in case when there are $\mathrm{m}+\mathrm{N}$ claims in the system as minimum, and $\mathrm{N}$ of them are in the memory. Such a queue exists if a newly arrived claim finds a minimum N claims waiting for service before this one.

At $\mathrm{N}=0$ we have a physical queue which is explicitly studied in the work [7]. In the system of GPSS World simulation modeling this characteristic has the name "a queue without zero inputs".

At $\mathrm{N}=1$ we have the so-called real queue; at $\mathrm{N}>1$ we have queues of higher orders.

Received: July 20, 2016

Published: September 10, 2016
(C) 2016 Academic Publications, Ltd.

url: www.acadpubl.eu 


\section{Real Queue}

Apparently, T. Saaty was the first to state the issue of real queues in his classical monograph [8]; it specified the value for the $\mathrm{M} / \mathrm{M} / \mathrm{m}$ system representing itself as an average number of demands which stay in the queue for some time to be served. Later on, this approach was developed in monographs [2] and [3] where characteristics of such queues for a number of QS basic models including a mixed type model (with expectation and refusals) and a model with a limited mean staying time of the claim in a queue waiting for service were systematized and examined. The monographs [2] and [3] proposed to call such queues as real queues. The physical sense defined in the meaning of [2], [3] and [8] of the real queue is that in this case a newly arrived into the system claim finds busy all service channels (all devices) and, at least, one more claim in the queue waiting for the service. Thus, the minimum mean length of a real queue (in case the intensity of an input stream of claims tends to zero) is unity but not zero as a general and well- studied mathematical queue has. As we see, the real queue in the meaning of [2], [3] and [8] is understood as the situation when there is at least one claim in the queue for service on a par.

The most general mathematical model of open multi-channel QS having $m$ service facilities of identical efficiency with exponentially distributed time of service was presented in the work of authors [5].The input demand stream in this case is a superposition of the arbitrary number of $h$ components each of which represents a Poisson stream of claims served in the queue. For each type of demands arriving in the system from $\mathrm{j}$-th source limitation for a queue length $\varepsilon_{j}$ acts, at the same time $\varepsilon_{0}<\varepsilon_{1}<\varepsilon_{2}<\cdots<\varepsilon_{h}$.

We will also use designations accepted in the work [5] in the present paper:

$$
\varepsilon_{0}=E_{0}=0 ; \varepsilon_{1}=E_{1} ; \varepsilon_{2}=E_{1}+E_{2} ; \cdots \varepsilon_{j}=\sum_{i=0}^{j} E_{i}=\sum_{i=1}^{j} E_{i} ; \text { a size-limited }
$$

queue (memory volume) for claims of the $\mathrm{j}$-th component;

$$
\Lambda_{0}=\sum_{j=0}^{h} \lambda_{j} ; \Lambda_{1}=\sum_{j=1}^{h} \lambda_{j} ; \Lambda_{2}=\sum_{j=2}^{h} \lambda_{j} ; \cdots \Lambda_{h}=\lambda_{h} ; \text { where } \lambda_{j} \text { claims stream }
$$

intensity of the $\mathrm{j}$-th component; $\mu$ claims service intensity by one service facility.

$$
R_{0}=\sum_{j=0}^{h} \rho_{j} ; R_{1}=\sum_{j=1}^{h} \rho_{j} ; R_{2}=\sum_{j=2}^{h} \rho_{j} ; \cdots R_{h}=\rho_{h} ; R_{i}=\frac{\Lambda_{i}}{\mu}, \text { where } \rho_{j} \text { is }
$$

the given claims stream intensity of the $j$-th component.

To calculate parameters of a physical queue on the basis of a universal mathematical model developed and presented in the work [5], it is necessary to rewrite a normalization condition in such a way as upon totaling in this condition to consider only those states of the system which correspond to a 
physical queue. As a result, we have

$$
\sum_{i=m+1}^{m+\varepsilon_{h}} P_{i}=1
$$

- normalization condition to calculate a real queue.

Substituting corresponding expressions for probabilities of steady states $P_{i}$ in the normalization condition presented above, and solving the obtained equation in regard to $P_{0}$, we will obtain the following expression of $\tilde{P}_{0}$ quantity used for a real queue calculation:

$$
\begin{gathered}
\tilde{P}_{0}=\left[\frac{R_{0}^{m}}{m !} \sum_{g=1}^{h} \prod_{j=0}^{g-1}\left(\frac{R_{j}}{m}\right)^{E_{j}} \times\right. \\
\times\left\{\begin{array}{l}
\frac{R_{g}}{m-R_{g}}\left(1-\left(\frac{R_{g}}{m}\right)^{E_{g}}\right), \quad R_{g} \neq m \\
E_{g}, \quad R_{g}=m
\end{array}\right\} ;
\end{gathered}
$$

Let's emphasize that in this case $\tilde{P}_{0}$ does not represent the probability of a zero state of the system $P_{0}$ any more, i.e. the probability of total claims absence in the system, which is not necessary in this calculation, but some coefficient of proportionality which can be even larger than unity. Only those probabilities values of corresponding states of $\tilde{P}_{i}$ system have physical sense in this case which, of course, are less than unity for all $i>m$ values.

Formulas for other probabilistic characteristics intended for real queue parameters calculation will be the same as when characteristics of the usual mathematical queue with replacement of $P_{0}$ for $\tilde{P}_{0}$ are calculated. We have

$$
\begin{gathered}
\tilde{P}_{i}=\left(\frac{R_{j+1}}{m}\right)^{i-m-\varepsilon_{j}} \prod_{g=0}^{j}\left(\frac{R_{g}}{m}\right)^{E_{g}} \frac{R_{0}^{m}}{m !} \tilde{P}_{0}, \\
m+\varepsilon_{j} \leq i \leq m+\varepsilon_{j+1}, \quad 0 \leq j \leq h-1
\end{gathered}
$$

- probabilities of various QS states in a steady state mode;

$$
\tilde{P}_{B i}=\prod_{g=0}^{i-1}\left(\frac{R_{g}}{m}\right)^{E_{g}} \frac{R_{0}^{m}}{m !} \tilde{P}_{0}\left\{\begin{array}{l}
\frac{m}{m-R_{i}}\left(1-\left(\frac{R_{i}}{m}\right)^{E_{i}}\right), \quad R_{i} \neq m \\
E_{i}, \quad R_{i}=m
\end{array}\right.
$$

- the basic probability for QS characteristics calculation; 


$$
\tilde{P}_{m+\varepsilon_{h}}=\prod_{g=1}^{h}\left(\frac{R_{g}}{m}\right)^{E_{g}} \frac{R_{0}^{m}}{m !} \tilde{P}_{0}
$$

- congestion probability of the system;

$$
\tilde{P}_{W}=\frac{1}{R_{0}} \sum_{i=1}^{h} R_{i} \tilde{P}_{B i}
$$

- probability of a newly arrived claim service expectation in a real queue.

In this case, the relative throughout capacity of the system is $\tilde{q}=\tilde{P}-\frac{R_{1}}{R_{0}} \tilde{P}_{m}$ the absolute throughout capacity is apparently, $\tilde{A}=\Lambda_{0} \tilde{q}$.

The moments of claims number waiting for service in a real queue are calculated according to the same scheme as for a mathematical queue; however, the probabilities with a tilde obtained with the corresponding normalization condition in view are inserted into corresponding formulas. A detailed conclusion of formulas for the moments of queue length is given in the work [5]. As a result, we have

- an average number of claims in a real queue

$$
\begin{aligned}
& \overline{\tilde{l}}=\sum_{i=1}^{h}\left\{\begin{array}{l}
\frac{R_{i}}{m-R_{i}}\left[\tilde{P}_{B i}-E_{i} \tilde{P}_{m+\varepsilon_{i}}\right], \quad R_{i} \neq m \\
\frac{E_{i}\left(E_{i}+1\right)}{2} \tilde{P}_{m+\varepsilon_{i-1}}, \quad R_{i}=m
\end{array}\right\}+ \\
& \sum_{i=2}^{h} \varepsilon_{i-1} \frac{R_{i}}{m} \tilde{P}_{B i}
\end{aligned}
$$

- the second initial moment of demands number in a real queue

$$
\begin{aligned}
\overline{\tilde{l}}^{2}= & \sum_{i=1}^{h}\left[\varepsilon_{i-1}^{2} \tilde{P}_{B i}+\left\{\frac{R_{i}}{m-R_{i}}\left(\frac{m+R_{i}}{m-R_{i}}+2 \varepsilon_{i-1}\right) \tilde{P}_{B i}-\right.\right. \\
& -\frac{m E_{i}}{m-R_{i}}\left(E_{i}+\frac{2 R_{i}}{m-R_{i}}+2 \varepsilon_{i-1}\right) \tilde{P}_{m+\varepsilon_{i}}, R_{i} \neq m \\
& \left.\left.\left(E_{i}-1\right) E_{i}\left(\frac{2 E_{i}-1}{6}+\varepsilon_{i-1}\right) \tilde{P}_{m+\varepsilon_{i}}, \quad R_{i}=m\right\}\right]+\varepsilon_{h}^{2} \tilde{P}_{m+\varepsilon_{h}} .
\end{aligned}
$$

The algorithm for calculation of claim servicing-waiting moments in a real queue is quite identical to the algorithm for calculation of claim servicingwaiting moments in a general mathematical queue presented in the work [5]. Corresponding probabilities with a tilde obtained with a new normalization condition in view have to be inserted instead of common probability characteristics (1). Besides, taking into account this circumstance it is necessary to redefine the relative throughout capacity of $\tilde{q}$ system. In this case, we have the 
following finite formulas for the claim servicing-waiting moments in the real queue for the QS universal model presented in the work [5]:

$$
\begin{aligned}
& \overline{\tilde{t}_{W}}=\frac{1}{\tilde{A}} \sum_{i=1}^{h}\left[\left\{\begin{array}{l}
\frac{R_{i}}{m-R_{i}}\left[\frac{R_{i}}{m} \tilde{P}_{B i}-E_{i} \tilde{P}_{m+\varepsilon_{i}}\right], R_{i} \neq m \\
\frac{E_{i}\left(E_{i}-1\right)}{2} \tilde{P}_{m+\varepsilon_{i-1}}, R_{i}=m
\end{array}\right\}+\frac{R_{i}}{m} \varepsilon_{i-1} \tilde{P}_{B i}\right] \\
& \overline{\tilde{t}_{W}^{2}}=\frac{1}{\tilde{A}}\left\{\begin{array}{l}
R_{1}\left[\frac{2\left(\frac{R_{1}}{m} \tilde{P}_{B 1}-E_{1} \tilde{P}_{m+E_{1}}\right)}{\mu\left(m-R_{1}\right)^{2}}-\frac{\left(E_{1}-1\right) E_{1} \tilde{P}_{m+E_{1}}}{m \mu\left(m-R_{1}\right)}\right], R_{1} \neq m \\
\frac{R_{1}^{2} \tilde{P}_{m}}{3 m^{3} \mu}\left(E_{1}-1\right) E_{1}\left(E_{1}+1\right), R_{1}=m
\end{array}\right\} \\
& +\frac{1}{\tilde{A}}\left\{\begin{array}{l}
\sum_{i=2}^{h} R_{i}\left[\frac{2\left(\tilde{P}_{B i}-E_{i} \tilde{P}_{m+\varepsilon_{i}}\right)}{\mu\left(m-R_{i}\right)^{2}}\left[1+\frac{\varepsilon_{i-1}-1}{m}\left(m-R_{i}\right)\right]+\right. \\
\sum_{i=2}^{h} \frac{R_{i}^{2} \tilde{P}_{m}}{3 m^{3} \mu} \prod_{g=0}^{i-1}\left(\frac{R_{g}}{R_{i}}\right)^{E_{g}} \times
\end{array}\right. \\
& \left.\begin{array}{ll}
\left.+\frac{\left(\varepsilon_{i-1}-1\right) \varepsilon_{i-1} \tilde{P}_{B i}}{m^{2} \mu}-\frac{E_{i}\left(E_{i}+1\right) \tilde{P}_{m+\varepsilon_{i}}}{m \mu\left(m-R_{i}\right)}\right], & R_{i} \neq m \\
\left.\left(\varepsilon_{i}+1\right)-\left(\varepsilon_{i-1}-1\right) \varepsilon_{i-1}\left(\varepsilon_{i-1}+1\right)\right], & R_{i}=m
\end{array}\right\} .
\end{aligned}
$$

\section{Higher Orders Queues}

Let's remind that an N-th order queue will be called the queue calculated in case when there are $\mathrm{m}+\mathrm{N}$ claims in the system as minimum, and $\mathrm{N}$ of them are in the memory. The general calculation algorithm of an $\mathrm{N}$-th order queue in case that $\mathrm{N}<\mathrm{h}$ is convenient to formulate using QS models with a full set of memories proposed in the work [6].

In this case, the normalization condition can be written as

$$
\sum_{i=m+N}^{m+h} P_{i}=1 .
$$

Then, probabilities of system steady states take the following form when a corresponding queue is calculated

$$
\tilde{P}_{0}=\left[\frac{R_{0}^{m}}{m !} \sum_{g=N}^{h} \prod_{j=1}^{g} \frac{R_{j}}{m}\right]^{-1}
$$




$$
\tilde{P}_{i}=\prod_{g=1}^{i-m} \frac{R_{g}}{m} \frac{R_{0}^{m}}{m !} \tilde{P}_{0}, \quad m+N \leq i \leq m+h ;
$$

- K-th order moments of claims number expecting service in the queue of $\mathrm{N}$-th order

$$
\tilde{l}_{N}^{(K)}=\sum_{i=m+N}^{m+h}(i-m)^{K} \tilde{P}_{i}
$$

- the relative throughout capacity of the system

$$
\tilde{q}=\frac{1}{R_{0}} \sum_{i=N+1}^{h} R_{i} \tilde{P}_{m+i-1} ;
$$

- waiting time distribution function of service by the claim in the queue of $\mathrm{N}$-th order

$$
\tilde{F}_{W N}(t)=1-\frac{1}{\tilde{q} R_{0}} \sum_{i=N+1}^{h} R_{i} \tilde{P}_{m+i-1} \sum_{j=0}^{i-N-1} B_{j}(t)
$$

where $B_{j}(t)=e^{-m \mu t} \frac{(m \mu t)^{j}}{j !}-$ the probability that $j$ claims are serviced by the system in time $t$;

- waiting service time distribution density by a claim in the queue of $\mathrm{N}$-th order

$$
\tilde{f}_{W N}(t)=\frac{d \tilde{F}_{W N}(t)}{d t} ;
$$

- K-th order moments of waiting service time by the claim in the queue of $\mathrm{N}$-th order

$$
\tilde{t}_{W N}^{(K)}=\int_{0}^{\infty} t^{K} \tilde{f}_{W N}(t) d t .
$$

Following the general scheme, it is possible to calculate queues parameters of any order for all private models of QS presented in the works [1], [4], [5] and [9]. 


\section{References}

[1] J.W. Cohen, Certain delay problems for a full availability trunk group loaded by two sources, Communication News, 16, No. 3 (1956), 105-113.

[2] A.P.Kirpichnikov Prikladnaya teoriya massovogo obsluzhivaniya [Applied queuing theory], Publishing office of KSU Publ., Kazan (2008), In Russian.

[3] A.P.Kirpichnikov Metody prikladnoy teorii massovogo obsluzhivaniya [Methods of applied queuing theory], Publishing office of KSU Publ., Kazan (2011), In Russian.

[4] A.P. Kirpichnikov, A.S. Titovtsev, Open systems of multicomponent flows differentiated service, Ciência e Técnica Vitivinícola, 29, No. 7 (2014), 108-122.

[5] A. Kirpichnikov, A. Titovtsev, Mathematical model of a queuing system with arbitrary quantity of sources and size-limited queue, International Journal of Pure and Applied Mathematics, 106, No. 2 (2016), 649-661.

[6] A. Kirpichnikov, A. Titovtsev, Mathematical model of open queuing system with full set of memories, International Journal of Pure and Applied Mathematics, 107, No. 1 (2016), 139-143.

[7] A. Kirpichnikov, A. Titovtsev, Physical and mathematical queues in the applied queuing theory, International Journal of Pure and Applied Mathematics, 108, No. 2 (2016), 409418.

[8] T.L. Saaty Elements of queueing theory with applications, McGRAW-HILL book company Inc., New York Toronto London (1961).

[9] H. Takagi, Explicit delay distribution in first-come first-served $\mathrm{M} / \mathrm{M} / \mathrm{m} / \mathrm{K}$ and $\mathrm{M} / \mathrm{M} / \mathrm{m} / \mathrm{K} / \mathrm{n}$ queues and mixed loss-delay system, International Journal of Pure and Applied Mathematics, 40, No. 2 (2007), 185-200. 
